OPEN ACCESS

Edited by:

Kiyoshi Itagaki,

Harvard Medical School, USA

Reviewed by:

Annapurna Nayak,

Brunel University London, UK

Cordula M. Stover,

University of Leicester, UK

${ }^{*}$ Correspondence:

Shaolin Shi

shaolinshi1001@yahoo.com

Specialty section:

This article was submitted to

Molecular Innate Immunity,

a section of the journal

Frontiers in Immunology

Received: 31 May 2016

Accepted: 25 January 2017

Published: 08 February 2017

Citation:

Xia H, Bao W and Shi S (2017)

Innate Immune Activity in

Glomerular Podocytes.

Front. Immunol. 8:122.

doi: 10.3389/fimmu.2017.00122

\section{Innate Immune Activity in Glomerular Podocytes}

\author{
Hong Xia ${ }^{1,2}$, Wenduona $\mathrm{Bao}^{1}$ and Shaolin Shi ${ }^{1 *}$ \\ ${ }^{1}$ National Clinical Research Center for Kidney Diseases, Jinling Hospital, Nanjing University School of Medicine, Nanjing, \\ China, ${ }^{2}$ Department of Nephrology, Zhejiang Provincial Hospital of Traditional Chinese Medicine, Hangzhou, China
}

Glomerular podocytes are specialized in structure and play an essential role in glomerular filtration. In addition, podocyte stress can initiate glomerular damage by inducing the injury of other glomerular cell types. Studies have shown that podocytes possess the property of immune cells and may be involved in adaptive immunity. Emerging studies have also shown that podocytes possess signaling pathways of innate immune responses and that innate immune responses often result in podocyte injury. More recently, mitochondrial-derived damage-associated molecular patterns (mtDAMPs) have been shown to play a critical role in a variety of pathological processes in cells. In the present mini-review, we summarize the recent advances in the studies of innate immunity and its pathogenic role in podocytes, particularly, from the perspective of mtDAMPs.

Keywords: innate immunity, mitochondrial DAMPs, podocyte injury, apoptosis, lupus nephropathy

Podocytes are highly specialized in structure and characterized by abundant foot processes between which slit diaphragms are formed and serve as part of glomerular filtration barrier. Podocyte homeostasis is critical for optimal glomerular filtration and podocyte injury or loss can initiate glomerular damage, possibly leading to glomerulosclerosis.

Podocytes exhibit features of immune cells. For example, podocytes express certain genes characteristic of immune cells, including MHC class II (1), B7-1 (CD80, a molecule required for T cell activation) (2), and FcRn (a receptor protein in antigen-presenting lymphocytes) (3). It has been demonstrated that podocytes can act as antigen-presenting cells as shown by their capabilities of phagocytosing and processing antigens and presenting them by forming peptide-MHC complexes, which can activate T cells (4). Importantly, this property of podocytes as antigen-presenting cells is involved in the development of nephritis as shown by amelioration of nephritis in the mice lacking MHC II specifically in podocytes in an anti-GBM nephritis model (4). Recently, increasing numbers of studies have shown that podocytes possess the components of innate immunity. The innate immune responses induced by various ligands have pathological role, leading to podocyte injury and podocytopathies. We will review the studies in this area and will discuss mitochondrial damageassociated molecular patterns (DAMPs) and their involvement in the innate immune activity in podocytes.

\section{INNATE IMMUNE RESPONSES IN PODOCYTES}

\section{Innate Immune System in Podocytes}

Podocytes express many genes required for innate immune responses. These genes include a number of pattern recognition receptors (PRRs) that sense both pathogen-associated molecular patterns (PAMPs, e.g., microbial pathogens) and DAMPs (e.g., endogenous molecules in an organism). In addition, the components of downstream signaling pathways are also present in podocytes. 


\section{Toll-Like Receptors (TLRs)}

Most members of the TLR family are detectable at mRNA level in isolated mouse glomeruli with TLR3 and TLR4 having the most abundant expression (5). Immunohistochemical staining showed that TLR4 protein is localized in podocytes. In cultured podocytes, the treatment with lipopolysaccharides (LPS), a PAMP ligand for TLR4, induced the expression of CCL2, CCL7, CXCL1, CXCL5, CCL3, CCL5, CXCL7, CXCL9, CXCL11, and CXCL13. Moreover, fibrinogen, a DAMP ligand of TLR4, also induced the expression of CCL2, CCL7, CXCL1, and CXCL5 (5). Consistently, nuclear factor kappa B (NFkB) signaling was found activated in podocytes that were treated with TLR ligands (6). Supportively, the essential components of TLR signaling in cultured podocytes, including MyD88, IRAK, TRAF6, etc., have also been detected (7). In patients with lupus nephritis, polyoma virus infection, or hemolytic uremic syndrome, TLR9 was found to be de novo expressed in podocytes and thought to be involved in immune response and inflammation in glomeruli (8-10).

\section{NLRP3 and Inflammasomes}

The major components of inflammasomes, NLRP3, ASC, and caspase 1, were found to be expressed in podocytes (11), and PAMP treatment was capable of upregulating the expression of NLRP3, pro-caspase 1 , posttranslational processing of pro-caspase 1 , and the release of IL-18 in podocytes (12). Cellular endogenous reactive oxygen species (ROS) was observed to induce the activation of NLRP 3 and formation of inflammasomes in mouse podocytes and glomeruli (13).

\section{Retinoic Acid-Inducible Gene 1 (RIG-I)}

Retinoic acid-inducible gene 1 belongs to the family of RIG-I-like helicases, which recognize viral RNA. It is expressed in podocytes and fully functional via downstream pathways, IRF3 (transcription factor interferon regulatory factor 3 ), and $\mathrm{NF \kappa B}$, leading to cytokines expression and podocyte injury (14).

\section{Receptor of Advanced Glycation Endproducts (RAGE)}

Receptor of advanced glycation endproducts is one of the PRRs and can use DAMPS as its ligands. These DAMPs include advanced glycation endproducts (AGE), high-mobility group box protein 1 (HMGB1), S100A proteins, etc. RAGE is expressed in podocytes and upregulated in diabetic nephropathy (DN) $(15,16)$, and mediate podocyte injury induced by AGE $(17)$. More recently, advanced oxidation protein products were also shown to be ligands of RAGE and capable of inducing podocyte apoptosis (18).

\section{Innate Immune Responses Are Associated with Podocyte Injury}

Although innate immune signaling pathways are present and can be activated in podocytes, there has been no study showing that the innate immune responses enable podocytes to eliminate pathogens in the body. Instead, a number of reports have described that innate immune responses lead to podocyte injury.
It was shown that injection of a TLR4 ligand, LPS, into mice induced proteinuria, accompanied by podocyte foot process effacement, in $24 \mathrm{~h}$ (2). This effect of LPS requires the expression of B7-1 (CD80) in podocytes because LPS does not induce proteinuria in B7-1 knockout mice (2). TLR3 and RIG-I were shown to mediate the effects of viruses on podocytes, leading to structural and functional disturbance of podocytes (14). This conclusion was further supported by the observation that the treatment with polyIC, a TLR3 ligand, caused proteinuria and changes in podocytes (19). TLR signaling is also involved in podocyte injury in DN by mediating the effect of serum LPS on podocytes (20).

Apoptosis is a major consequence of podocyte injury. Intrinsic caspase 3 pathway was shown to be responsible for podocyte apoptosis. However, it has just been shown that it is caspase 1, but not caspase 3, that mediates podocyte injury, at least, in DN, and that this process involves NLRP3/inflammasomal pathway (21). Involvement of NLRP3/inflammasomes was also demonstrated in other podocyte injury model (22). This finding is unexpected and surprising and has led to paradigm shift regarding the mechanism underlying podocyte death. It is important to further investigate how NLRP3/inflammasomes are activated and what the responsible ligands are.

\section{MITOCHONDRIAL DAMPs (mtDAMPs)}

Mitochondria are believed to have evolved from ancient bacteria through endosymbiogenesis. They, therefore, have their own DNA and protein synthesis machinery. Mitochondrial DNA (mtDNA) and the proteins synthesized in mitochondria are both different from nuclear DNA (nDNA) and the proteins synthesized on ER.

\section{Formyl Peptides}

Like bacteria, mitochondria use formylmethionine (methionine attached with a formyl group) for the initiation of protein synthesis, leaving the synthesized proteins formyl modified. During evolution, eukaryotic organisms developed formyl-peptide receptors that recognize bacterial formyl peptides, leading to innate immune responses and bacterial clearance. It is known that mitochondrial formyl peptides are still capable of inducing innate immune responses (23).

\section{Mitochondrial DNA}

Mitochondrial DNA retains the features of bacterial DNA, including CpG unmethylation. Like bacterial DNA, which acts on TLR9 to induce innate inflammatory response or NLRP3 to produce proinflammatory cytokine in immune cells $(24,25)$, mtDNA has been shown to act on TLR9 and NLRP3 similarly in macrophages (26-28).

\section{Mitochondrial Transcription Factor A (Tfam)}

Mitochondrial transcription factor A is a nuclear gene-encoded protein that is functionally and structurally homologous to HMGB1, a known endogenous danger molecule, and has been shown to act as a danger molecule as well. It can work with 
unmethylated CpG DNA to act on RAGE and induce response of plasmacytoid dendritic cells, leading to release of TNF- $\alpha$ (29, 30). In addition, recombinant Tfam treatment can induce TNF- $\alpha$ expression in cultured macrophages, as well as inflammatory responses in animals (31).

\section{mtDAMPs INDUCE INNATE IMMUNE RESPONSES AND INJURY IN PODOCYTES}

Podocytes possess many PRRs that are functional as discussed above. Particularly, podocytes express TLR9 and NLRP3 that can use mtDNA as ligand, as well as RAGE that can use Tfam as ligand. These suggest that mtDAMPs may act on podocytes to activate innate immune pathways.

\section{Endogenous mtDNA and TLR9 Mediate Puromycin Aminonucleoside (PAN)-Induced Podocyte Injury}

Puromycin aminonucleoside has recently been shown to be capable of inducing mtDNA translocation to lysosomes in which mtDNA acts as ligand to stimulate TLR9 in podocytes, thereby enhancing proapoptotic NFkB and p38 MAPK signaling, inflammatory cytokine expression, and apoptosis level $(7,32)$. Nevertheless, mtDNA may also act through NLPR3/inflammasomes/caspase 1 pathway in the experimental system because of the presence of the NLRP3-caspase 1 pathway in podocytes (11). As already mentioned earlier, caspase 1 may be more important than caspase 3 in podocyte apoptosis, at least, in the condition of diabetes (21).

\section{mtDNA-NLRP3 Interaction May Underlie Podocyte Injury in DN}

We speculate that mtDNA-NLRP3 interaction may be an important mechanism underlying podocyte injury/apoptosis in DN. It is known that high glucose can induce ROS in podocytes, as well as apoptosis of the cells (33), and ROS can induce mtDNA oxidative damage in podocytes (34). Importantly, oxidized mtDNA has been shown to be more potent in activating NLRP3, inflammasomes, and apoptosis in macrophages (27). Together with the observation that caspase 1 , but not caspase 3 , mediates podocyte injury in DN (21), these studies strongly suggest that high glucose or hyperglycemia may cause podocyte injury or death through an innate immune pathway involving NLRP3 in diabetes.

\section{AGE-RAGE Interaction May Also Contribute to Podocyte Injury in DN}

Innate immune responses can also be induced by AGE through interaction with RAGE and lead to podocyte injury. Under diabetic condition, it was found that a high level of AGE (e.g., glycated albumin) can cause podocyte apoptosis via RAGE, leading to innate immune response and podocyte apoptosis (35).

\section{mtDAMPs May Play an Important} Role in Lupus Nephropathy (LN)

Systemic lupus erythematosus (SLE) is a type of autoimmune disease in which the immune system of the patient becomes hyperactive and attacks normal tissues, including kidney. In the development of SLE and LN, mtDAMPs likely play a role described as follow.

\section{mtDNA/TLR9 May Be Involved in the Development of SLE and LN}

In SLE-prone MRL-Fas(lpr) mice, the treatment with DNA derived from bacteria or CpG ODN facilitated the development of SLE and LN in the mice through activating TLR9 in B cells $(36,37)$. The treated mice manifested an increase of DNA autoantibodies, cytokines, or chemokines in circulation, and infiltration of immune cells in tissues, including kidney.

In addition to B cells, mtDNA-TLR9 may also facilitate SLE development through plasmacytoid predendritic cells (PDCs) as there has been a study showing that viral DNA or the DNA in the immune complexes can stimulate PDCs through TLR9 to express and secrete IFN- $\alpha$, a cytokine that is involved in antidsDNA antibody production (38). Meanwhile, mtDNA has been shown to have antigenicity that contributes to the formation of anti-dsDNA antibodies. Interestingly, oxidized form of mtDNA exhibited an enhanced antigenicity compared to that not oxidized (39). Additionally, the autoantibodies in SLE patients are reactive to mtDNA and have a higher affinity with mtDNA than nDNA $(40,41)$. Consistently, the level of anti-mitochondrial antibody is correlated with the development of SLE (42). These observations suggest that mtDNA and TLR9 are involved in the development of SLE and LN.

\section{mtDNA/TLR9 May Contribute to}

\section{Podocyte Injury in LN}

Puromycin aminonucleoside is capable of inducing IFN- $\alpha$ expression in podocytes via mtDNA and TLR9 interaction (7). In addition, several studies have shown that TLR9 is de novo expressed in podocytes in a proportion of SLE patients (8-10). This raises a possibility that podocytes are an alternative source of IFN- $\alpha$ and other cytokines that are involved in SLE and LN development. SLE is characterized by the production of anti-double stranded (ds) DNA antibodies, which bind to dsDNA to form immune complexes (ICs). Such DNA-containing ICs are capable of stimulating plasmacytoid PDCs to express IFN- $\alpha$ through TLRs, including TLR9 (38), it is thus reasonable to speculate that the anti-dsDNA antibodies can also bind mtDNA to form ICs. Since lupus nephritis is pathologically characterized by the deposition of the ICs in glomeruli, we speculate that the mtDNA in the ICs may be transported to podocytes through endocytosis and then either reach endolysosomes to activate the de novo expressed TLR9 or act on NLRP3/inflammasomes, resulting in podocyte injury/apoptosis and IFN- $\alpha$ and proinflammatory IL-1 production thereby facilitating LN development. To prove this hypothesis, it would be important to examine whether the ICs in lupus contain mtDNA. 
As having mentioned above that TLR9 de novo expression in podocytes occurs in some of the patients of LN with urinary protein level of $0.3-0.5 \mathrm{~g} / 24 \mathrm{~h}$ or a negative urinary protein (9), it would be interesting to investigate the consequences of TLR9 de novo expression in podocytes in the condition. Hu and colleague have recently reported that podocytopathy in the LN patients with nephrotic range of proteinuria can be divided into two types, MCD/mesangial proliferation and FSGS, which differ in pathology, tubular lesion, and treatment outcome (43). Whether TLR9 expression underlies the difference of the two types of lupus podocytopathy warrants further investigation. One feasible approach for the study could be to perform immunohistochemical staining of TLR9 with renal biopsies in a cohort of LN consisting of patients with MCD/mesangial proliferation and FSGS; and then test whether TLR9 de novo expression in podocytes correlates with the patients with FSGS, which manifests with podocyte injury and loss in contrast with MCD.

\section{CIRCULATING mIDAMPs AND PODOCYTE INFLAMMATION AND INJURY}

Proteinuria is frequently observed in the patients with trauma and burning and the resulting sterile systemic inflammation response syndrome (SIRs) (44-47). Interestingly, elevation of circulating mtDNA level has also been observed in the patients (48-52). It is thus possible that mtDNA in circulation may play a pathologic role in proteinuria development through damaging podocytes. Moreover, since the increase of mtDNA in circulation should be accompanied by other mtDAMPs, e.g., Tfam, it is also possible that these mtDAMPs may act in concert to damage podocytes. One study has shown that injection of mtDAMPs into rats via tail can cause transient mild proteinuria several hours following the

\section{REFERENCES}

1. Coers W, Brouwer E, Vos JT, Chand A, Huitema S, Heeringa P, et al. Podocyte expression of MHC class I and II and intercellular adhesion molecule-1 (ICAM-1) in experimental pauci-immune crescentic glomerulonephritis. Clin Exp Immunol (1994) 98:279-86. doi:10.1111/j.1365-2249.1994.tb06138.x

2. Reiser J, von Gersdorff G, Loos M, Oh J, Asanuma K, Giardino L, et al. Induction of B7-1 in podocytes is associated with nephrotic syndrome. J Clin Invest (2004) 113:1390-7. doi:10.1172/JCI20402

3. Akilesh S, Huber TB, Wu H, Wang G, Hartleben B, Kopp JB, et al. Podocytes use FcRn to clear IgG from the glomerular basement membrane. Proc Natl Acad Sci U S A (2008) 105:967-72. doi:10.1073/pnas.0711515105

4. Goldwich A, Burkard M, Olke M, Daniel C, Amann K, Hugo C, et al. Podocytes are non-hematopoietic professional antigen-presenting cells. J Am Soc Nephrol (2013) 24:906-16. doi:10.1681/ASN.2012020133

5. Banas MC, Banas B, Hudkins KL, Wietecha TA, Iyoda M, Bock E, et al. TLR4 links podocytes with the innate immune system to mediate glomerular injury. J Am Soc Nephrol (2008) 19(4):704-13. doi:10.1681/ASN.2007040395

6. Shimada M, Ishimoto T, Lee PY, Lanaspa MA, Rivard CJ, Roncal-Jimenez CA, et al. Toll-like receptor 3 ligands induce CD80 expression in human podocytes via an NF-אB-dependent pathway. Nephrol Dial Transplant (2012) 27(1):81-9. doi:10.1093/ndt/gfr271

7. Bao W, Xia H, Liang Y, Ye Y, Lu Y, Xu X, et al. Toll-like receptor 9 can be activated by endogenous mitochondrial DNA to induce podocyte apoptosis. Sci Rep (2016) 6:22579. doi:10.1038/srep22579

8. Papadimitraki ED, Tzardi M, Bertsias G, Sotsiou E, Boumpas DT. Glomerular expression of toll-like receptor-9 in lupus nephritis but not in normal kidneys: injection (32). This little effect of mtDAMPs on podocytes may be due to the fact that TLR9 is not expressed in podocytes of normal rats, thus precluding the action of mtDNA. Thus, the mtDAMPs other than mtDNA might be responsible for the mild proteinuria in the rats. Whether a long-term high level of mtDAMPs in circulation would cause more severe podocyte injury, proteinuria and even glomerulosclerosis would be interesting to explore.

\section{CONCLUDING REMARKS}

The available studies have shown that podocytes possess many features of immune cells of both adaptive and innate immunity. As non-immune cells, podocytes are usually injured by the innate immune responses induced by PAMPs or DAMPs (including mtDAMPs). Therefore, further studies of innate immune responses in podocytes would provide more insights into the mechanism underlying podocyte injury, likely leading to improved therapeutics and diagnostics for podocytopathies.

\section{AUTHOR CONTRIBUTIONS}

HX: collected literature and wrote manuscript; WB: collected literature and discussed the manuscript; SS: chose the topic, collected literature, and wrote manuscript.

\section{FUNDING}

This work was supported by the grants from the National Natural Science Foundation of China (81370827, 91442104), Natural Science Foundation of Jiangsu Province (BK20131324), Jiangsu Innovation Talent Program (2011-32) and Science and Technology Program of Zhejiang Traditional Chinese Medicine (2017ZA053).

implications for the amplification of the inflammatory response. Lupus (2009) 18(9):831-5. doi:10.1177/0961203309103054

9. Machida $H$, Ito $S$, Hirose $T$, Takeshita F, Oshiro H, Nakamura T, et al. Expression of toll-like receptor 9 in renal podocytes in childhood-onset active and inactive lupus nephritis. Nephrol Dial Transplant (2010) 25(8):2530-7. doi:10.1093/ndt/gfq058

10. Batsford S, Duermueller U, Seemayer C, Mueller C, Hopfer H, Mihatsch M. Protein level expression of toll-like receptors 2, 4 and 9 in renal disease. Nephrol Dial Transplant (2011) 26(4):1413-6. doi:10.1093/ndt/gfq752

11. Zhang C, Boini KM, Xia M, Abais JM, Li X, Liu Q, et al. Activation of Nod-like receptor protein 3 inflammasomes turns on podocyte injury and glomerular sclerosis in hyperhomocysteinemia. Hypertension (2012) 60:154-62. doi:10.1161/HYPERTENSIONAHA.111

12. Solini A, Menini S, Rossi C, Ricci C, Santini E, Blasetti Fantauzzi C, et al. The purinergic $2 \mathrm{X} 7$ receptor participates in renal inflammation and injury induced by high-fat diet: possible role of NLRP3 inflammasome activation. J Pathol (2013) 231(3):342-53. doi:10.1002/path.4237

13. Abais JM, Xia M, Li G, Gehr TW, Boini KM, Li PL. Contribution of endogenously produced reactive oxygen species to the activation of podocyte NLRP3 inflammasomes in hyperhomocysteinemia. Free Radic Biol Med (2014) 67:211-20. doi:10.1074/jbc.M114.567537

14. Yamashita M, Millward CA, Inoshita H, Saikia P, Chattopadhyay S, Sen GC, et al. Antiviral innate immunity disturbs podocyte cell function. J Innate Immun (2013) 5(3):231-41. doi:10.1159/000345255

15. Abel M, Ritthaler U, Zhang Y, Deng Y, Schmidt AM, Greten J, et al. Expression of receptors for advanced glycosylated end-products in renal disease. Nephrol Dial Transplant (1995) 10(9):1662-7. 
16. Tanji N, Markowitz GS, Fu C, Kislinger T, Taguchi A, Pischetsrieder M, et al. Expression of advanced glycation end products and their cellular receptor RAGE in diabetic nephropathy and non-diabetic renal disease. J Am Soc Nephrol (2000) 11(9):1656-66. doi:10.1681/ASN.2007101109

17. Guo J, Ananthakrishnan R, Qu W, Lu Y, Reiniger N, Zeng S, et al. RAGE mediates podocyte injury in adriamycin-induced glomerulosclerosis. J Am Soc Nephrol (2008) 19(5):961-72. doi:10.1681/ASN.2007101109

18. Zhou LL, Cao W, Xie C, Tian J, Zhou Z, Zhou Q, et al. The receptor of advanced glycation end products plays a central role in advanced oxidation protein products-induced podocyte apoptosis. Kidney Int (2012) 82(7):759-70. doi:10.1038/ki.2012.184

19. Ishimoto T, Shimada M, Gabriela G, Kosugi T, Sato W, Lee PY, et al. Toll-like receptor 3 ligand, polyIC, induces proteinuria and glomerular CD80, and increases urinary CD80 in mice. Nephrol Dial Transplant (2013) 28(6):143946. doi:10.1093/ndt/gfs543

20. Saurus P, Kuusela S, Lehtonen E, Hyvönen ME, Ristola M, Fogarty CL, et al. Podocyte apoptosis is prevented by blocking the toll-like receptor pathway. Cell Death Dis (2015) 6:e1752. doi:10.1038/cddis.2015.125

21. Shahzad K, Bock F, Al-Dabet MM, Gadi I, Kohli S, Nazir S, et al. Caspase-1, but not caspase-3, promotes diabetic nephropathy. J Am Soc Nephrol (2016) 27(8):2270-5. doi:10.1681/ASN.2015060676

22. Abais JM, Xia M, Li G, Chen Y, Conley SM, Gehr TW, et al. Nod-like receptor protein 3 (NLRP3) inflammasome activation and podocyte injury via thioredoxin-interacting protein (TXNIP) during hyperhomocysteinemia. J Biol Chem (2014) 289(39):27159-68. doi:10.1074/jbc.M114.567537

23. Le Y, Murphy PM, Wang JM. Formyl-peptide receptors revisited. Trends Immunol (2002) 23(11):541-8. doi:10.1016/S1471-4906(02)02316-5

24. Hemmi H, Takeuchi O, Kawai T, Kaisho T, Sato S, Sanjo H, et al. A tolllike receptor recognizes bacterial DNA. Nature (2000) 408(6813):740-5. doi:10.1038/35047123

25. Muruve DA, Pétrilli V, Zaiss AK, White LR, Clark SA, Ross PJ, et al. The inflammasome recognizes cytosolic microbial and host DNA and triggers an innate immune response. Nature (2008) 452(7183):103-7. doi:10.1038/ nature 06664

26. Nakahira K, Haspel JA, Rathinam VA, Lee SJ, Dolinay T, Lam HC, et al. Autophagy proteins regulate innate immune responses by inhibiting the release of mitochondrial DNA mediated by the NALP3 inflammasome. Nat Immunol (2011) 12:222-30. doi:10.1038/ni.1980

27. Shimada K, Crother TR, Karlin J, Dagvadorj J, Chiba N, Chen S, et al. Oxidized mitochondrial DNA activates the NLRP3 inflammasome during apoptosis. Immunity (2012) 36:401-14. doi:10.1016/j.immuni.2012.01.009

28. Horng T. Calcium signaling and mitochondrial destabilization in the triggering of the NLRP3 inflammasome. Trends Immunol (2014) 35:253-61. doi:10.1016/j.it.2014.02.007

29. Julian M, Shao G, Bao S, Knoell D, Papenfuss T, VanGundy Z, et al. Mitochondrial transcription factor A (TFAM) serves as a danger signal by augmenting plasmacytoid dendritic cell responses to DNA. J Immunol (2012) 189(1):433-43. doi:10.4049/jimmunol.1101375

30. Julian MW, Shao G, Vangundy ZC, Papenfuss TL, Crouser ED. Mitochondrial transcription factor A, an endogenous danger signal, promotes TNF $\alpha$ release via RAGE- and TLR9-responsive plasmacytoid dendritic cells. PLoS One (2013) 8(8):e72354. doi:10.1371/journal.pone

31. Chaung W, Wu R, Ji Y, Dong W, Wang P. Mitochondrial transcription factor A is a proinflammatory mediator in hemorrhagic shock. Int J Mol Med (2012) 30(1):199-203. doi:10.3892/ijmm.2012.959

32. He J, Lu Y, Xia H, Liang Y, Wang X, Bao W, et al. Circulating mitochondrial DAMPs are not effective inducers of proteinuria and kidney injury in rodents. PLoS One (2015) 10(4):e0124469. doi:10.1371/journal.pone.0124469

33. Susztak K, Raff AC, Schiffer M, Böttinger EP. Glucose-induced reactive oxygen species cause apoptosis of podocytes and podocyte depletion at the onset of diabetic nephropathy. Diabetes (2006) 55(1):225-33. doi:10.2337/ diabetes.55.01.06.db05-0894

34. Casalena G, Krick S, Daehn I, Yu L, Ju W, Shi S, et al. Mpv17 in mitochondria protects podocytes against mitochondrial dysfunction and apoptosis in vivo and in vitro. Am J Physiol Renal Physiol (2014) 306(11):F1372-80. doi:10.1152/ ajprenal.00608.2013

35. Chuang P, Yu Q, Fang W, Uribarri J, He JC. Advanced glycation endproducts induce podocyte apoptosis by activation of the FOXO4 transcription factor. Kidney Int (2007) 72(8):965-76. doi:10.1038/sj.ki.5002456
36. Anders H, Vielhauer V, Eis V, Linde Y, Kretzler M, Perez, et al. Activation of toll-like receptor-9 induces progression of renal disease in MRL-Fas(lpr) mice. FASEB J (2004) 18(3):534-46. doi:10.1096/f.03-0646fje

37. Pawar RD, Patole PS, Ellwart A, Lech M, Segerer S, Schlondorff D, et al. Ligands to nucleic acid-specific toll-like receptors and the onset of lupus nephritis. J Am Soc Nephrol (2006) 17(12):3365-73. doi:10.1681/ASN.2006030263

38. Barrat F, Meeker T, Gregorio J, Chan JH, Uematsu S, Akira S, et al. Nucleic acids of mammalian origin can act as endogenous ligands for toll-like receptors and may promote systemic lupus erythematosus. J Exp Med (2005) 202(8):1131-9. doi:10.1084/jem.20050914

39. Alam K, Moinuddin, Jabeen S. Immunogenicity of mitochondrial DNA modified by hydroxyl radical. Cell Immunol (2007) 247(1):12-7. doi:10.1016/ j.cellimm.2007.06.007

40. Reimer G, Rubin RL, Kotzin BL, Tan EM. Anti-native DNA antibodies from autoimmune sera also bind to DNA in mitochondria. J Immunol (1984) 133(5):2532-6.

41. Ivanova VV, Khaiboullina SF, Cherenkova EE, Martynova EV, Nevzorova TA, Kunst MA, et al. Differential immuno-reactivity to genomic DNA, RNA and mitochondrial DNA is associated with auto-immunity. Cell Physiol Biochem (2014) 34(6):2200-8. doi:10.1159/000369663

42. Wang H, Li T, Chen S, Gu Y, Ye S. Neutrophil extracellular trap mitochondrial DNA and its autoantibody in systemic lupus erythematosus and a proof-ofconcept trial of metformin. Arthritis Rheumatol (2015) 67(12):3190-200. doi:10.1002/art.39296

43. Hu W, Chen Y, Wang S, Chen H, Liu Z, Zeng C, et al. Clinical-morphological features and outcomes of lupus podocytopathy. Clin J Am Soc Nephrol (2016) 11(4):585-92. doi:10.2215/CJN.06720615

44. Shakespeare PG, Coombes EJ, Hambleton J, Furness D. Proteinuria after burn injury. Ann Clin Biochem (1981) 18:353-60. doi:10.1177/000456328101 800606

45. Gosling P, Sutcliffe AJ. Proteinuria following trauma. Ann Clin Biochem (1986) 23:681-5. doi:10.1177/000456328602300610

46. De Gaudio AR, Spina R, Di Filippo A, Feri M. Glomerular permeability and trauma: a correlation between microalbuminuria and injury severity score. Crit Care Med (1999) 27:2105-8. doi:10.1097/00003246-19991000000004

47. Axelsson J, Mahmutovic I, Rippe A, Rippe B. Loss of size selectivity of the glomerular filtration barrier in rats following laparotomy and muscle trauma. Am J Physiol Renal Physiol (2009) 297:F577-82. doi:10.1152/ajprenal.00246. 2009

48. Lam NY, Rainer TH, Chiu RW, Joynt GM, Lo YM. Plasma mitochondrial DNA concentrations after trauma. Clin Chem (2004) 50:213-6. doi:10.1373/ clinchem.2003.025783

49. Simmons JD, Lee YL, Mulekar S, Kuck JL, Brevard SB, Gonzalez RP, et al. Elevated levels of plasma mitochondrial DNA DAMPs are linked to clinical outcome in severely injured human subjects. Ann Surg (2013) 258:591-8. doi:10.1097/SLA.0b013e3182a4ea46

50. Gu X, Yao Y, Wu G, Lv T, Luo L, Song Y. The plasma mitochondrial DNA is an independent predictor for post-traumatic systemic inflammatory response syndrome. PLoS One (2013) 8:e72834. doi:10.1371/journal.pone. 0072834

51. Chou CC, Fang HY, Chen YL, Wu CY, Siao FY, Chou MJ. Plasma nuclear DNA and mitochondrial DNA as prognostic markers in corrosive injury patients. Dig Surg (2008) 25:300-4. doi:10.1159/000152846

52. Yamanouchi S, Kudo D, Yamada M, Miyagawa N, Furukawa H, Kushimoto S. Plasma mitochondrial DNA levels in patients with trauma and severe sepsis: time course and the association with clinical status. J Crit Care (2013) 28:1027-31. doi:10.1016/j.jcrc.2013.05.006

Conflict of Interest Statement: The authors declare that the research was conducted in the absence of any commercial or financial relationships that could be construed as a potential conflict of interest.

Copyright ( $(2017$ Xia, Bao and Shi. This is an open-access article distributed under the terms of the Creative Commons Attribution License (CC BY). The use, distribution or reproduction in other forums is permitted, provided the original author(s) or licensor are credited and that the original publication in this journal is cited, in accordance with accepted academic practice. No use, distribution or reproduction is permitted which does not comply with these terms. 